\title{
The Moon Meets All Requirements of the IAU Definition for "Planet"
}

\author{
David G. Russell \\ Science Department, Owego Free Academy, Owego, NY, USA \\ Email: russelld@oacsd.org
}

How to cite this paper: Russell, D.G. (2017) The Moon Meets All Requirements of the IAU Definition for "Planet". International Journal of Astronomy and Astrophysics, 7, 291-302. https://doi.org/10.4236/ijaa.2017.74024

Received: November 1, 2017

Accepted: December 15, 2017

Published: December 18, 2017

Copyright $\odot 2017$ by author and Scientific Research Publishing Inc. This work is licensed under the Creative Commons Attribution International License (CC BY 4.0).

http://creativecommons.org/licenses/by/4.0/

(c) (i) Open Access

\begin{abstract}
The Earth-Moon system has often been characterized as having some characteristics of a "double planet" system. It is demonstrated that while the orbital barycenter of the Earth-Moon pair lies inside the radius of the Earth, the Moon does meet all three requirements of the IAU definition for "planet" and therefore the Moon can correctly be identified as the Solar System's $9^{\text {th }}$ planet. In order to avoid confusion by this development it is necessary to add definitions for "double planet", "double dwarf planet", "satellite planet", and "satellite" to complement the International Astronomical Union definitions for "planet" and "dwarf planet". The Earth-Moon system meets the requirements of a "double planet" system while the Pluto-Charon system meets the requirements of a "double dwarf planet" system. In order to extrapolate sub-stellar taxonomy to exoplanetary systems, general formation mechanisms (star-like gas collapse in molecular clouds vs. proto-planetary disk formation) should be included in the definitions for the various classes of sub-stellar mass bodies.
\end{abstract}

\section{Keywords}

Moon, Earth, Planet, Dwarf Planet, Double Planet, Satellite, Rogue Planet, Brown Dwarf

\section{Introduction}

Due to the large radius of the Moon relative to the Earth, late $19^{\text {th }}$ to late $20^{\text {th }}$ century textbooks frequently suggested that the Earth-Moon system, in some respects, can be characterized as a double planet [1]-[7]. While the terms "satellite" and "double planet" have not been formally defined by the International Astronomical Union (IAU), it has been proposed that a pair be considered a "double planet" if the satellite is massive enough that the orbital barycenter lies between the pair and outside the body of the primary [8]. The mutual orbital 
barycenter of the Earth-Moon system lies inside the body of the Earth and therefore the system does not qualify as a double planet according to this definition.

The IAU definition for "planet" requires that a body meet three criteria to qualify as a planet. The body must: 1) orbit the sun; 2) be massive enough to self-gravitate into a spherical shape; and 3) dynamically dominate or "clear" its orbital zone. While the Earth-moon system does not meet the proposed orbital barycenter definition for a double planet, it can be verified that the Moon does meet all three requirements for planethood as defined by the IAU even though it has not been included on the IAU list of planets. The omission of a body, the Moon, that qualifies as a planet according to the criteria of the IAU definition for "planet" can be addressed with one or more of the following changes: 1) adding the Moon to the list of planets as the Solar System's $9^{\text {th }}$ planet; or 2) refining the definition of planet with additional criteria the Moon does not meet; or 3) adding definitions for "double planet" and "satellite" to the IAU taxonomy.

This paper is organized as follows: Evidence that the Moon meets all three criteria of the IAU planet definition is discussed in Sections 2, 3, and 4. In Section 5 the implications of the Moon as a planet are discussed and new dynamical definitions are proposed to complement the IAU "planet" and "dwarf planet" definitions. Section 6 is the conclusion.

\section{The Shape of the Moon}

The IAU "planet" and "dwarf planet" definitions both require a body have enough mass to self-gravitate into a spherical shape. The minimum radius an icy body can have and still self-gravitate into a spherical shape is $200-225 \mathrm{~km} \mathrm{[9]} \mathrm{[10].}$ The smallest spherical icy moons in the Solar System have masses of $3.7-6.6 \times$ $10^{19} \mathrm{~kg}$ (Table 1). For rocky bodies the minimum radius to attain a spherical shape is $\sim 300 \mathrm{~km}$ [9]. The smallest unambiguously spherical rocky body in the Solar System is the dwarf planet Ceres with a mass of $9.47 \times 10^{20} \mathrm{~kg}$. The mass of the Moon is therefore at least 80 times greater than the minimum mass a rocky body needs to attain a spherical shape.

The equatorial diameter $(1738.1 \mathrm{~km})$ and polar diameter $(1736.0 \mathrm{~km})$ of the Moon differ by only $0.12 \%$ demonstrating the Moon has a spherical shape. In addition, recent studies indicate the Moon is a differentiated body with dense solid and liquid layers in the core and a mantle underlying the lunar crust [11] [12]. Both the shape and internal structure of the Moon therefore indicate an evolved planetary body in a near hydrostatic equilibrium state.

\section{The Moon's Orbital Clearing Capacity}

The IAU planet definition requires that to qualify as a planet a body must be dynamically dominant and have "cleared the neighborhood around its orbit". Dynamical dominance has been defined as the ability of a body to clear the neighborhood of its orbit within a Hubble time or the host stars main sequence 
Table 1. Planets, dwarf planets, and spherical satellites of the solar system.

\begin{tabular}{|c|c|c|c|}
\hline Planetary Body & Mass $(\mathrm{kg})^{*}$ & Radius $(\mathrm{km})^{*}$ & Density $\mathrm{g} \cdot \mathrm{cm}^{-3 *}$ \\
\hline Jupiter & $1.90 \times 10^{27}$ & 69911 & 1.326 \\
\hline Saturn & $5.68 \times 10^{26}$ & 58232 & 0.687 \\
\hline Neptune & $1.02 \times 10^{26}$ & 24622 & 1.638 \\
\hline Uranus & $8.68 \times 10^{25}$ & 25631 & 1.270 \\
\hline Earth & $5.97 \times 10^{24}$ & 6371 & 5.513 \\
\hline Venus & $4.87 \times 10^{24}$ & 6052 & 5.243 \\
\hline Mars & $6.40 \times 10^{23}$ & 3390 & 3.934 \\
\hline Mercury & $3.30 \times 10^{23}$ & 2440 & 5.427 \\
\hline Ganymede & $1.48 \times 10^{23}$ & 2631 & 1.94 \\
\hline Titan & $1.30 \times 10^{23}$ & 2575 & 1.88 \\
\hline Callisto & $1.08 \times 10^{23}$ & 2410 & 1.83 \\
\hline Io & $8.93 \times 10^{22}$ & 1822 & 3.53 \\
\hline Moon & $7.35 \times 10^{22}$ & 1738 & 3.34 \\
\hline Europa & $4.80 \times 10^{22}$ & 1561 & 3.01 \\
\hline Triton & $2.10 \times 10^{22}$ & 1353 & 2.06 \\
\hline Eris & $1.67 \times 10^{22}$ & 1163 & 2.50 \\
\hline Pluto & $1.31 \times 10^{22}$ & 1151 & 2.05 \\
\hline Titania & $3.4 \times 10^{21}$ & 789 & 1.66 \\
\hline Oberon & $2.9 \times 10^{21}$ & 761 & 1.56 \\
\hline Rhea & $2.3 \times 10^{21}$ & 764 & 1.23 \\
\hline Iapetus & $1.8 \times 10^{21}$ & 736 & 1.08 \\
\hline Charon & $1.55 \times 10^{21}$ & 604 & 1.68 \\
\hline Ariel & $1.3 \times 10^{21}$ & 599 & 1.59 \\
\hline Umbriel & $1.2 \times 10^{21}$ & 585 & 1.46 \\
\hline Dione & $1.1 \times 10^{21}$ & 562 & 1.48 \\
\hline Ceres & $9.47 \times 10^{20}$ & 476 & 2.09 \\
\hline Tethys & $6.18 \times 10^{20}$ & 533 & 0.97 \\
\hline Enceladus & $1.08 \times 10^{20}$ & 252 & 1.61 \\
\hline Miranda & $6.6 \times 10^{19}$ & 236 & 1.21 \\
\hline Mimas & $3.75 \times 10^{19}$ & 198 & 1.15 \\
\hline
\end{tabular}

*Planetary data is from the NASA solar system exploration webpage available at http://solarsystem.nasa.gov/planets/index.cfm

lifetime [8] [13] [14]. The Earth-Moon orbital zone was primarily cleared by the Earth. However, if the Earth-Moon system is a double planet, the Moon should also be massive enough to clear the Earth-Moon system's orbital zone, as suggested by [8]. 
Soter [13] proposed that a body is dynamically dominant in its orbital zone if its mass is at least 100 times greater than the mass of all other bodies currently sharing its orbital zone. The "planetary discriminant" $(\mu)$ is defined as the mass of a candidate planetary body $(M)$ divided by the mass of all bodies crossing its orbital zone $(m)$ [13]. According to the data provided in [13] the mass of all bodies sharing the orbital zone of Mercury, Venus and Earth is approximately $3.6 \times$ $10^{18} \mathrm{~kg}$. The Moon's mass is $7.35 \times 10^{22} \mathrm{~kg}$ and therefore the Moon has a planetary discriminant value of $2.0 \times 10^{4}$ and meets the definition for a cleared orbit proposed by [13].

Based upon the recent formulation of Margot [14] a body needs a minimum mass of $7.4 \times 10^{21} \mathrm{~kg}$ to clear the Earth's orbital zone within the main sequence lifetime of the Sun (Table 2). The Moon's mass is therefore 10 times greater than the minimum mass needed to clear the Earth-Moon system orbital zone and massive enough to clear orbital zones at least as distant as Jupiter (Table 2).

As orbital clearing has been defined by [8] [13] [14], the Moon, by itself, exceeds the minimum mass needed to clear the Earth-Moon orbital zone.

\section{The Moon's Orbital Primary}

The first requirement of the IAU planet definition is that a planet must orbit the Sun. Therefore, planets have a heliocentric orbit and the strongest gravitational force experienced by a planet will come from the Sun and not from other planets or attendant satellites. For satellites of planets, a comparison between the gravitational force exerted by the primary planet on the satellite and the gravitational force exerted by the Sun on the satellite can be expressed as a tug-of-war (TOW) ratio $\mathrm{fp} / \mathrm{fs}$ [15] [16] where "fp" is the gravitational force exerted by the planet on the satellite and "fs" is the gravitational force exerted by the Sun on the same satellite. The TOW ratio indicates how many times greater the pull of the primary

Table 2. Minimum mass to clear planetary orbits in the solar system.

\begin{tabular}{ccc}
\hline Body & Mass to clear $(\oplus)$ & Mass to clear $(\mathrm{kg})^{*}$ \\
\hline Mercury & $4.23 \times 10^{-4}$ & $2.5 \times 10^{21}$ \\
Venus & $8.58 \times 10^{-4}$ & $5.1 \times 10^{21}$ \\
Earth & $1.23 \times 10^{-3}$ & $7.4 \times 10^{21}$ \\
Mars & $1.98 \times 10^{-3}$ & $1.2 \times 10^{22}$ \\
Ceres & $4.00 \times 10^{-3}$ & $2.4 \times 10^{22}$ \\
Jupiter & $7.95 \times 10^{-3}$ & $4.7 \times 10^{22}$ \\
Saturn & $1.56 \times 10^{-2}$ & $9.3 \times 10^{22}$ \\
Uranus & $3.46 \times 10^{-2}$ & $2.1 \times 10^{23}$ \\
Neptune & $5.70 \times 10^{-2}$ & $3.4 \times 10^{23}$ \\
Pluto & $7.80 \times 10^{-2}$ & $4.7 \times 10^{23}$ \\
Eris & $1.40 \times 10^{-1}$ & $8.4 \times 10^{23}$ \\
\hline
\end{tabular}

*Value calculated from $\mathrm{M}_{\text {clear }}=\mathrm{M}_{\text {body }}(\oplus) / \Pi$ and data in Table 1 of Margot [14]. 
planet is on the satellite than the Sun's pull on the same satellite. The TOW values for the spherical satellites of Jupiter, Saturn, Uranus, and Neptune range from 45 to 24,600 [15], indicating these large spherical moons are firmly within the gravitational control of their primary planet.

The TOW value for the Moon is 0.46 [15] [16] which indicates that the Sun's gravitational pull on the Moon is greater than the gravitational pull exerted on the Moon by the Earth. This important fact was noted at least as early as 1836 by Sir John Herschel [17]:

"If we execute the numerical calculation in the case before us, we shall find 2.209:1 for the proportion in which the intensity of the force which retains the Earth in its orbit round the Sun actually exceeds that by which the moon is retained in its orbit about the Earth."

Herschel's ratio can be converted to a TOW value of 0.452 , which is very close to the modern value of 0.46 . The implication of the greater gravitational pull exerted by the Sun on the Moon than the Earth on the Moon was noted by Whipple [18]:

"Strictly speaking, the Earth perturbs the motion of the Moon about the Sun because the Sun actually attracts the Moon with a force nearly twice as great as the attraction of the Earth on the Moon."

As a consequence of the stronger pull exerted on the Moon by the Sun, the Moon's orbit is heliocentric and always concave toward the Sun and not the Earth. In order to illustrate the Moon's phases, modern introductory astronomy textbooks provide a simplified version of the Moon's orbit as a circular figure in geocentric motion around the Earth [2] [19] [20] [21]. These representations provide an incorrect impression of a lunar orbit always concave toward the Earth. However, the correct shape of the Moon's orbit has been provided in description and diagram (Figure 1) in a number of introductory astronomy textbooks dating back to the mid-19 $9^{\text {th }}$ century [3] [6] [7] [17] [18], and was recently reanalyzed by [16]. The Moon's orbit never loops around the Earth and is always concave toward the Sun [16].

The TOW value and orbital figure for the Moon both demonstrate that the Moon's orbital primary is the Sun, not the Earth. Therefore, while the Moon is generally characterized as the Earth's satellite, the Moon's orbit is that of a planet orbiting the Sun.

\section{Discussion}

\subsection{Dynamical Classes for Spherical Solar System Bodies}

In the preceding sections it was demonstrated that the Moon meets all three criteria necessary for planethood as described in the IAU planet definition: 1) the Moon has a heliocentric orbit; 2) the Moon has a spherical shape; and 3) the Moon has sufficient mass to dynamically clear its orbital zone. The Moon therefore legitimately qualifies as the $9^{\text {th }}$ planet of the Solar System and the Earth-Moon system is a double planet pair. 


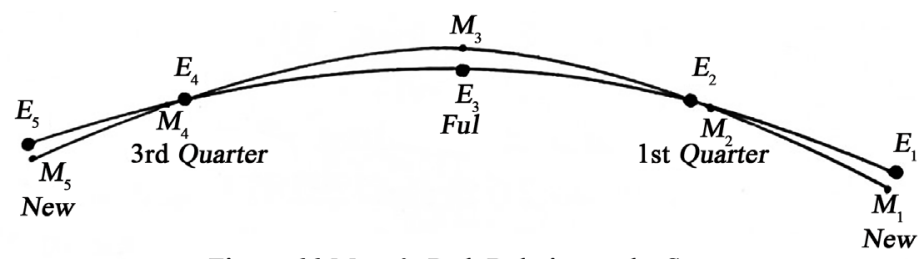

Figure 66-Moon's Path Relative to the Sun

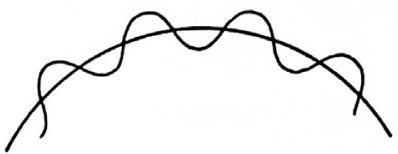

Figure 67

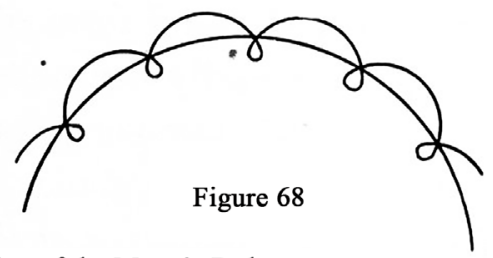

Figure 68

Erroneous Representation of the Moon's Path

Figure 1. Image showing correct and incorrect illustrations of the Moon's orbit from Young's Manual on Astronomy [7]. Figure 66 in the image correctly represents the Moon's orbital path always concave toward the sun. Figure 67 incorrectly represents the Moon's orbit with a shorter period and always concave toward the Earth. Figure 68 incorrectly represents the Moon completing loops around the Earth.

There are several important challenges this conclusion presents. First, having established a definition for "planet" in 2006, it is important that the IAU keep the list of planets updated to include all bodies that meet the criteria of the planet definition. With the parameters established by the IAU's "planet" definition, there is no formal criterion that justifies exclusion of the Moon from the list of planets unless the IAU passes a modification to the current planet definition. Second, by adding the Moon to the list of planets a taxonomic challenge is created in that satellites are also collectively referred to as "moons" and the body from which this name was generalized is not actually part of the "moon" class.

One reason for this dilemma is that while the IAU has formal definitions for spherical bodies that clear their orbit (planets), and spherical bodies that do not clear their orbit (dwarf planets), the IAU has not provided a definition resulting in a distinct category for spherical satellites such as Io, Europa, Ganymede, Callisto, Titan, and Triton. These spherical satellites are larger than the dwarf planets, and two have a larger radius than the planet Mercury. In addition, the IAU has not provided a definition for the circumstances under which a pair of bodies qualify as a double planet or double dwarf planet rather than a planet-satellite pair.

The following definitions are recommended to resolve these gaps in taxonomy:

Double planet: a pair of bodies orbiting the Sun, with each having enough mass to dynamically clear the orbit and self-gravitate into a spherical shape, and also meeting one or both of the following criteria: 1) the orbital barycenter lies outside the radius of the larger body in the pair; or 2) the Sun, rather than the larger planet in the pair, is the primary for the orbit of the smaller body in the pair. Both bodies in a double planet pair are planets.

Double dwarf planet: a pair of bodies orbiting the Sun in an orbit that is not 
dynamically cleared, with both massive enough to self-gravitate into a spherical shape, and also meeting one or both of the following criteria: 1) the orbital barycenter lies outside the radius of the larger body in the pair; or 2) the Sun-rather than the larger dwarf planet in the pair-is the primary for the orbit of the smaller body in the pair. Both bodies in a double dwarf planet pair are dwarf planets.

Binary planet-dwarf planet: a pair of bodies orbiting the Sun in an orbit that is dynamically cleared, with both massive enough to self-gravitate into a spherical shape, but with the smaller in the pair having insufficient mass to clear the orbit, and also meeting one or both of the following criteria: 1) the orbital barycenter lies outside the radius of the larger body in the pair; or 2) the Sun, rather than the planet in the pair, is the primary for the orbit of the smaller body in the pair.

Satellite planet or spherical satellite: a body massive enough to self-gravitate into a spherical shape with a primary orbit around a planet or dwarf planet.

Satellite: a body with insufficient mass to self-gravitate into a spherical shape that has a primary orbit around a planet, dwarf planet, or small solar system body.

These definitions, in combination with the IAU "planet" and "dwarf planet" definitions, provide a more complete taxonomy for the dynamical circumstances of spherical sub-stellar bodies in the Solar System. Spherical bodies that dynamically dominate, and clear, their orbit are planets or double planet pairs. Spherical bodies that do not dynamically dominate, or clear, their orbit are dwarf planets or double dwarf planet pairs. Spherical bodies that orbit a more massive spherical body are satellite planets. A census of spherical Solar System bodies meeting these classes is provided in Table 3. Spherical trans-Neptunian bodies not yet included on the IAU dwarf planet list are not listed in Table 3.

Table 3. Spherical sub-stellar bodies of the solar system.

\begin{tabular}{ccc}
\hline Planets & Dwarf planets & Satellite planets (planet) \\
\hline Mercury & Ceres & Io (Jupiter) \\
Venus & Pluto \& Charon ${ }^{\mathrm{b}}$ & Europa (Jupiter) \\
Earth \& Moon & Eris & Ganymede (Jupiter) \\
Mars & Haumea & Callisto (Jupiter) \\
Jupiter & Makemake & Titan (Saturn) \\
Saturn & & Rhea (Saturn) \\
Uranus & Iapetus (Saturn) \\
Neptune & Dione (Saturn) \\
& Tethys (Saturn) \\
& & Enceladus (Saturn) \\
& Mimas (Saturn) \\
& Titania (Uranus) \\
& Oberon (Uranus) \\
& Umbriel (Uranus) \\
& Ariel (Uranus) \\
& Miranda (Uranus) \\
& Triton (Neptune) \\
\hline
\end{tabular}

Notes: $\mathrm{a}$-double planet pair; $\mathrm{b}$-double dwarf planet pair. 
It is important to note that definitions similar to those provided above have been suggested in various forms by other authors. For example, Stern \& Levison [8] proposed a definition for "double planet" that only included the barycenter requirement. The circumstances of the Moon demonstrate that a double planet can also occur when both bodies in a pair sharing a common orbital barycenter have orbits that are concave toward the Sun, and for which the Sun is pulling with more gravitational force on each body than either body's gravitational pull on the other. Runyon et al. [22] proposed calling spherical satellites "moon planets" but the classification of the Moon as a planet makes the special designation "satellite planets" preferable.

\subsection{Exoplanets and Brown Dwarfs}

The IAU planet and dwarf planet definitions only apply to the Solar System as they state that a planet must orbit "the Sun". The IAU definitions can be extended to exoplanetary systems by changing "the Sun" to "a star". Margot [14] found that the Kepler and non-Kepler exoplanets discovered to date all have a mass exceeding the minimum mass required to clear their orbital zone within the main sequence lifetime of their host stars.

An interesting problem is presented by the existence of free-floating planetary mass bodies [23]. These bodies are not orbiting a star and therefore do not meet the requirements of the IAU planet definition. Some of these bodies may be planets formed in a proto-planetary disk around a star that subsequently have been ejected from their orbit. Others may have formed from star-like gas collapse mechanisms and represent the low mass end of the stellar formation process-brown dwarfs.

The IAU has defined brown dwarfs as sub-stellar bodies that exceed the deuterium burning limit, or 13 Jupiter masses, regardless of formation mechanism. However, based upon formation mechanisms, brown dwarfs form by gas collapse mechanisms in molecular clouds whereas planets form in a proto-planetary disk around a forming star [24] [25]. Star-like gas collapse mechanisms can form bodies below the deuterium burning limit, and bodies formed in a proto-planetary disk can exceed the deuterium burning limit [25]-[30]. Mass-radius and mass-density relationships from 0.3 to 60 Jupiter masses lack any distinguishing feature at $13 \mathrm{Ju}$ piter masses to indicate any physical significance to deuterium burning [31] [32]. Given these problems it has been suggested that formation mechanism, not the deuterium burning limit, should be used to define the difference between giant planets and brown dwarfs [25].

The issue of formation mechanism must be resolved in order to consistently extend the IAU planet definition to exoplanetary circumstances. Are free floating planetary mass bodies planets or brown dwarfs? Is a spherical body orbiting a brown dwarf a satellite or a planet? Is a body exceeding the deuterium burning limit that formed in a proto-planetary disk a brown dwarf or a planet? These questions cannot be answered without deciding how formation mechanism should be incorporated into the definitions. 
An objection to including formation mechanism in the definitions for "brown dwarf" and "planet" is that it may be difficult to assign a formation history to individual sub-stellar bodies and our understanding of formation mechanisms is certainly incomplete [14]. However, brown dwarfs were originally identified as star formation products that do not acquire sufficient mass to begin hydrogen burning on the stellar main sequence [33]. The distinction between a brown dwarf and a giant planet is tightly linked to formation mechanisms [25].

The following definitions are recommended for extending the taxonomy of spherical sub-stellar bodies to additional exoplanetary circumstances not found in the Solar System:

Brown dwarf: a gaseous, sub-stellar mass body, formed by star-like gas collapse mechanisms, with insufficient mass to enable core hydrogen fusion.

Rogue planet: a free floating, sub-stellar mass body, formed in a protoplanetary disk, with sufficient mass to self-gravitate into a spherical shape, which has been ejected from its circumstellar orbit, and therefore no longer orbits a star or brown dwarf.

Since brown dwarfs form in a star-like manner, spherical bodies orbiting a brown dwarf that formed in the brown dwarf s proto-planetary disk are planets or dwarf planets. The IAU definitions for planet and dwarf planet should be modified to include the requirement that these bodies formed in a proto-planetary disk.

\section{Conclusions}

In this paper it has been noted that the Moon is a spherical body that is capable of clearing its orbit, and has a primary orbit around the Sun rather than the Earth. Therefore, the Moon meets all three criteria the IAU established for identifying a body as a planet and should be recognized as the $9^{\text {th }}$ planet of the Solar System. In recognizing the Moon as a planet, it becomes necessary to complete the taxonomy started with the IAU "planet" and "dwarf planet" definitions by providing clear definitions for "double planet", "double dwarf planet", and "satellite planet".

The Earth-Moon system is a "double planet" and the Pluto-Charon system is a "double dwarf planet". Recognizing the spherical satellites (e.g. Ganymede, Ti$\tan )$ as the special class "satellite planet" corrects the lack of a special designation to distinguish these important spherical planetary bodies from the much more numerous non-spherical satellites.

Using the IAU "planet" and "dwarf planet" definitions, along with the definitions provided in this paper, results in the following numbers for spherical Solar System bodies (Table 3): 9 planets, 6 dwarf planets, and 17 satellite planets. The number of planets, dwarf planets, and satellite planets can be expected to grow as new data confirms the mass and orbital traits of additional trans-Neptunian bodies and attendant spherical satellites.

In extending the IAU definitions to exoplanetary systems and free floating planetary mass bodies, it is helpful to include formation mechanism in the definition 
to distinguish brown dwarfs from giant planets. Brown dwarfs are formed by star-like gas collapse mechanisms. Planets, dwarf planets, and spherical moons are formed in a proto-planetary disk. Free floating sub-stellar mass bodies will therefore be "rogue planets" if they are formed in a proto-planetary disk and were ejected from their circumstellar orbit. If the free floating sub-stellar body is formed by gas collapse in molecular clouds, it is a brown dwarf.

Finally, it is worth noting that some disagreement over the IAU "planet" and "dwarf planet" definitions persists. The new definitions presented here may help resolve the disagreement in several advantageous ways. First, the definitions presented do not require any significant changes to the IAU definitions but instead provide definitions that cover additional circumstances. Second, by establishing a definition- "satellite planet"-for spherical satellites, the geophysical importance of all spherical sub-stellar bodies is formally recognized and allows the revised classification of Pluto to "dwarf planet" to be set into a more complete context. Third, by establishing clear definitions for "double planet" and "double dwarf planet" Pluto's companion Charon can be correctly recognized as another dwarf planet in the Solar System.

\section{Acknowledgements}

This research has made use of NASA's Astrophysics Data System Bibliographic Services. I would like to thank the anonymous referee for helpful suggestions for improving this paper.

\section{References}

[1] Baker, R.H. (1959) Astronomy. 7th Edition, D. Van Nostrand Company Inc., New York.

[2] Kaler, J.B. (1994) Astronomy! Harper Collins College Publishers, New York.

[3] McLaughlin, D.B. (1961) Introduction to Astronomy. Houghton Mifflin Company, Boston.

[4] Payne-Gaposchkin, C. (1954) Introduction to Astronomy. Prentice Hall Inc., New York.

[5] Russell, H.N, Dugan, R.S. and Steward, J.Q. (1926) Astronomy I: The Solar System. Ginn and Company, Boston.

[6] Young, C.A. (1889) A Text-Book of General Astronomy. Ginn \& Company, Boston.

[7] Young, C.A. (1902) Manual of Astronomy. Ginn \& Company, New York.

[8] Stern, S.A. and Levison, H.F. (2002) Regarding the Criteria for Planethood and Proposed Planetary Classification Schemes. In: Rickman, H., Ed., Highlights of Astronomy 12: 205-213, Astronomical Society of the Pacific, San Francisco. https://doi.org/10.1017/S1539299600013289

[9] Lineweaver, C. and Norman, M. (2010) The Potato Radius: A Lower Minimum Size for Dwarf Planets. Preprint, arXiv:1004.1091.

[10] Tancredi, G. (2010) Physical and Dynamical Characteristics of Icy "Dwarf Planets" (Plutoids) Icy Bodies of the Solar System. Proceedings of the International Astronomical Union, IAU Symposium, 263, 173-185. 
[11] Weber, R.C., Lin, P.-Y., Garnero, E.J., Williams, Q. and Lognonné, P. (2011) Seismic Detection of the Lunar Core. Science, 331, 309-312. https://doi.org/10.1126/science.1199375

[12] Williams, J.G., Konopliv, A.S., Boggs, D.H., Park, R.S., Yuan, D.-N., Lemoine, F.G., Goossens, S., et al. (2014) Lunar Interior Properties from the GRAIL Mission. Journal of Geophysical Research, 119, 1546-1578. https://doi.org/10.1002/2013JE004559

[13] Soter, S. (2006) What Is a Planet? The Astronomical Journal, 132, 2513-2519. https://doi.org/10.1086/508861

[14] Margot, J.-L. (2015) A Quantitative Criterion for Defining Planets. The Astronomical Journal, 150, 185-192. https://doi.org/10.1088/0004-6256/150/6/185

[15] Asimov, I. (1975) Just Mooning Around. In: Asimov on Astronomy, Anchor Press/ Doubleday, New York.

[16] Legault, R.J. (2013) Moon Loops and Dumbbells-The Most Curious Moon of All. Journal of the Royal Astronomical Society of Canada, 107, 65-68.

[17] Herschel, J. (1836) A Treatise on Astronomy. Carey, Lea, \& Blanchard, Philadelphia.

[18] Whipple, F.L. (1941) Earth, Moon and Planets. The Blakiston Company, Philadelphia.

[19] Bless, R.C. (1996) Discovering the Cosmos. University Science Books, Sausilito.

[20] Chaisson, E. and McMillan, S. (2008) Astronomy Today. 6th Edition, Pearson-Addison Wesley, San Francisco.

[21] Schneider, S.E. and Arny, T.T. (2015) Pathways to Astronomy. 5th Edition, McGraw-Hill Education, New York.

[22] Runyon, K.D., Stern, S.A., Lauer, T.R., Grundy, W., Summers, M.E. and Singer, K.N. (2017) A Geophysical Planet Definition. 48th Lunar and Planetary Science Conference, LPI Contribution No. 1964, Article ID: 1448.

[23] Lucas, P.W. and Roche, P.F. (2000) A Population of Very Young Brown Dwarfs and Free-Floating Planets in Orion. Monthly Notices of the Royal Astronomical Society, 314, 858-864. https://doi.org/10.1046/j.1365-8711.2000.03515.x

[24] Kumar, S. (2003) The Bottom of the Main Sequence and Beyond: Speculations, Calculations, Observations, and Discoveries (1958-2002). IAUS, 211, 3-12. https://doi.org/10.1017/S0074180900210176

[25] Chabrier, G., Johansen, A., Janson, M. and Rafikov, R. (2014) Giant Planet and Brown Dwarf Formation. In: Planets, V.I., Beuther, H., Klessen, R., Dullemond, C. and Henning, T. (Eds.), Protostars, University of Arizona Press, Tucson, 619-642. https://doi.org/10.2458/azu_uapress_9780816531240-ch027

[26] Lucas, P., Weights, D., Roche, P. and Riddick, F. (2006) Spectroscopy of Planetary Mass Brown Dwarfs in Orion. Monthly Notices of the Royal Astronomical Society: Letters, 373, L60-L64. https://doi.org/10.1111/j.1745-3933.2006.00244.x

[27] Bihain, G., Rebolo, R., Zapatero Osorio, M.R., Béjar, V.J.S., Villó-Pérez, I., Díaz-Sánchez, A., Pérez-Garrido, A., Caballero, J.A., Bailer-Jones, C.A.L., Barrado y Navascués, D., Eislöffel, J., Forveille, T., Goldman, B., Henning, T., Martín, E.L. and Mundt, R. (2009) Candidate Free-Floating Super-Jupiters in the Young Sigma Orionis Open Cluster. Astronomy \& Astrophysics, 506, 1169-1182. https://doi.org/10.1051/0004-6361/200912210

[28] Caballero, J.A., Béjar, V.J.S., Rebolo, R., Eislöffel, J., Zapatero Osorio, M.R., Mundt, R., Barrado Y Navascués, D., Bihain, G., Bailer-Jones, C.A.L., Forveille, T. and Martín, 
E.L. (2007) The Substellar Mass Function in Sigma Orionis. II. Optical, Near-Infrared and IRAC/Spitzer Photometry of Young Cluster Brown Dwarfs and Planetary-Mass Objects. Astronomy \& Astrophysics, 470, 903-918.

https://doi.org/10.1051/0004-6361:20066993

[29] Ma, B. and Ge, J. (2014) Statistical Properties of Brown Dwarf Companions: Implications for Different Formation Mechanisms. Monthly Notices of the Royal Astronomical Society, 439, 2781-2789. https://doi.org/10.1093/mnras/stu134

[30] Mulders, G.D., Paardekooper, S.-J., Panić, O., Dominik, C., van Boekel, R. and Ratzka, T. (2013) Planet or Brown Dwarf? Inferring the Companion Mass in HD 100546 from the Wall Shape using Mid-Infrared Interferometry. Astronomy \& Astrophysics, 557, 68-78. https://doi.org/10.1051/0004-6361/201220930

[31] Hatzes, A. and Rauer, H. (2015) A Definition for Giant Planets Based on the Mass-Density Relationship. The Astrophysical Journal Letters, 810, L25-L29. https://doi.org/10.1088/2041-8205/810/2/L25

[32] Chen, G. and Kipping, D. (2017) Probabilistic Forecasting of the Masses and Radii of Other Worlds. The Astrophysical Journal, 834, 17-30. https://doi.org/10.3847/1538-4357/834/1/17

[33] Kumar, S. (1963) The Structure of Stars of Very Low Mass. The Astrophysical Journal, 137, 1121-1125. https://doi.org/10.1086/147589 